\title{
Formulations and Features of Person Selection in Organization Personnel Training Based on Supernetwork
}

\author{
Wei Sun ${ }^{1}$, Chunhui $\mathrm{Huo}^{2}$ and Yang $\mathrm{Yu}^{*}$ \\ ${ }^{1,2}$ Business School, Liaoning University, Shenyang, 110136, China, Institute of \\ Systems Engineering, Northeastern University, Shenyang, 110819, China \\ lnuhappysw@126.com,iwuzetian@126.com,yuyang@ise.neu.edu.cn
}

\begin{abstract}
To investigate the reasonable method for selecting persons in organization personnel training, our study focuses on the formulation of personnel selection in organization personnel training based on knowledge supernetwork. First, this paper states the process of constructing organization training supernetwork and formulates the model for selecting single person. Subsequently, we establish the model for selecting multiple persons based on training knowledge supernetwork and analyze the complexity and the shortcomings in detail. Third, to improve the accuracy of personnel selection, we build the model for selecting multiple persons based on step-by-step method and analyze the complexity. Final, the proposed models are verified by an application case.
\end{abstract}

Keywords: personnel training; supernetwork; single person selection; multiple person's selection; formulation

\section{Introduction}

With the advent of the knowledge economy, knowledge has become one of the most important organizational resources [1] and a key factor in organization. When an organization wants to supplement knowledge or improve knowledge structure, talent recruiting and personnel training often are considered [2]. Owing to the simplicity, personnel training is usually adopted. In personnel training, managers need to overcome the following problems: 1) how to determine organization needing knowledge (i.e., training content); and 2) how to select reasonable person(s) to be trained?

Up to now, there are few researches about the two problems. Yu et al. [3] proposed a method for selecting one person to train based on knowledge supernetwork. The main procedure can be summarized as following: 1) the knowledge supernetwork of organization personnel training needs to be constructed; 2) knowledge of the person based on the knowledge supernetwork is expressed; 3) organization needing knowledge is determined based on the knowledge supernetwork; 4) the similarity between organization needing knowledge and each personnel's knowledge is calculated; and 5) the person with the maximum similarity is selected to train.

The method proposed by $\mathrm{Yu}$ et al. [3] not only improves organization knowledge structure, but also shortens training time. That is because the person with the highest similarity to organization needing knowledge (i.e., training content) is trained. The shortcoming of the method [3] is that it can only select one person to train. For an organization, training multiple persons simultaneously is necessary. Therefore, our study focuses on formulating the models for multiple person's selection for organization personnel training and clarifying the complexities and features.

The reminder of this paper is organized as follows: Section 2 reviews the research status and introduces important methods used in the research. Section 3 formulates the model for selecting single person by quantifying the method [3] and states the shortcoming of selecting single person. Subsequently, we propose the model for 
selecting multiple persons. In section 4 , to solve the shortcoming of selecting multiple persons, we formulate the model for selecting multiple persons based on step-by-step method and analyze the complexity. Section 5 verifies our proposed models by an application case. Section 6 gives the conclusions and future problems.

\section{Personnel Training and Knowledge Supernetwork}

\subsection{Personnel Training}

Personnel training, also known as talent training [4], plays an important role in human resource management. In addition, personnel training is an effective method to enhance human resource level and to increase personnel knowledge and capacity.

In summary, most previous studies on personnel training focus on the importance of personnel training, objectives of personnel training and personnel training modes [5]. In addition, the procedure of person selection in personnel training generally includes [6] 1) determination of evaluation index, 2) evaluation of each person according to index system, and 3) selection of the person with highest evaluation score. The evaluation index includes [7] field, title, gender, age, and knowledge. Field, title, gender and age can be easily determined. However, personnel knowledge is difficult to be expressed and evaluated, even though knowledge is very significant for supplementing and improving organization knowledge structure.

To overcome the difficulty in expressing and evaluating personnel knowledge, Yu et al. [3] proposed a method for selecting one person to train based on knowledge supernetwork. The main contribution can be descried as: 1) knowledge supernetwork of organization personnel training is constructed to express a personnel's knowledge; 2) organization needing knowledge is determined based on the knowledge supernetwork; 3) the similarity between organization needing knowledge and the personnel's knowledge is calculated; and 4) the person with the maximum similarity is selected for being trained.

\subsection{Knowledge Supernetwork}

Knowledge supernetwork, as a kind of supernetwork [8], refers to the supernetwork containing multiple knowledge networks [9]. Knowledge supernetwork has been used to study the characteristics of knowledge system and to solve real problems. Xi et al. [10] used knowledge supernetwork to integrate different types of knowledge resources. Xi et al. [11] used knowledge supernetwork to analyze the influence of person loss on organizational knowledge structure. Sun et al. [12] studied the construction method of innovation team based on knowledge supernetwork. Sun et al. [13] investigated the method for recruiting talent based on knowledge supernetwork. In the research, knowledge supernetwork is used to select persons to be trained. We use the two following methods based on the knowledge supernetwork: expression of personnel knowledge and knowledge similarity degree calculation [14].

The procedure of constructing personnel training knowledge supernetwork and related methods used in this research is described as follows.

2.2.1. File Preprocessing: File preprocessing is to transfer the file containing knowledge elements into the file (such as TXT file) which can be operated easily by computer.

2.2.2. Knowledge Element Mining: After file preprocessing, we can mine knowledge elements using knowledge mining tools. Knowledge mining tools are utilized to mine the concept-knowledge elements. So, common text mining tools can be used to mine 
knowledge elements. The detailed knowledge mining methods and tools can be found in $[9,10,15]$.

Construction of Knowledge Supernetwork for Organization Personnel Training: By using the personnel information and knowledge elements mined from files, we can construct the knowledge supernetwork for personnel training, as shown in Figure 1. Based on the knowledge supernetwork, we can express personnel knowledge, determine organization needing knowledge, and calculate the similarity between organization needing knowledge and the personnel's knowledge.

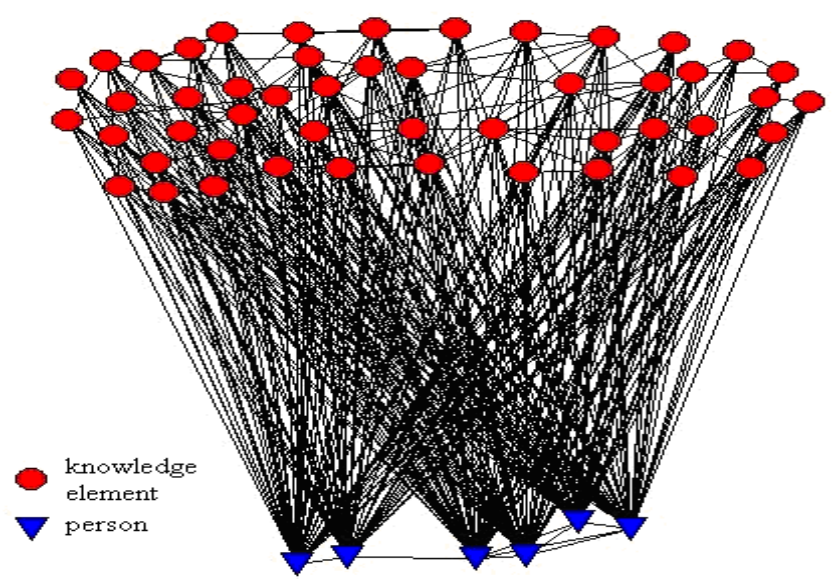

Figure 1. Knowledge Supernetwork of Organization Personnel Training

2.2.4. Expression of the Personnel's Knowledge: In Figure 1, the personnel set can be donated as $S P=\left\{P_{1}, P_{2}, \ldots, P_{n}\right\}$, where knowledge of $P_{i}$ can be expressed as:

$$
P_{i}=\vec{p}_{i}=\left(v_{i k}\right) 1 \times n=\left[v_{i 1}, v_{i 2}, \ldots, v_{i n}\right],
$$

where

$$
v_{i k}=\left\{\begin{array}{l}
t, k e_{k} \text { is contained in } P_{i} \\
0, k e_{k} \text { is not contained in } P_{i}
\end{array} .\right.
$$

If knowledge element $k e_{k}$ is not contained in $P_{i}$, then $v_{i k}=0$; otherwise, $v_{i k}=t$, where $t$ denotes the frequency of $k e_{i}$ appearing in $P_{i}$.

2.2.5. Organization Needing Knowledge: The method for determining the knowledge required by organization can be found in [3]. Assuming that organization needing knowledge $(O N K)$ is expressed as:

$$
O N K=\left[k e_{1}, k e_{2}, \ldots, k e_{t}\right]
$$

2.2.6. Simulation between Organization Needing Knowledge and the Personnel's Knowledge: All knowledge in all persons $(A K)$ can be expressed as:

$$
A K=\left[k e_{1}, k e_{2}, \ldots, k e_{l}\right]
$$

To produce a standard knowledge element vector $(K)$, we merge $O N K$ and $A K$.

$$
K=O N K \bigcup A K=\left[k e_{1}, k e_{2}, \ldots, k e_{t}\right] \cup\left[k e_{1}, k e_{2}, \ldots, k e_{l}\right]=\left[k e_{1}, k e_{2}, \ldots, k e_{n}\right]
$$

Based on the standard vector $(K)$, weight of the knowledge element $k e_{k}$ in $P_{i}$ can be calculated as [16, 17]: $W_{k e k}=t f \times i d f . t f=\frac{v_{i k}}{\sum_{k=1}^{n} v_{i k}}$, where $v_{i k}$ is the frequency $k e_{k}$ appearing 
in $P_{i}$ and $\sum_{k=1}^{n} v_{i k}$ denotes the total number of knowledge elements in $P_{i}$. $i d f=\lg \frac{|S P+O N G|}{\left|(S P+O N G)_{k e k}\right|}$, where $|S P+O N K|$ equals $|S P|+1$ (ONK is thought as a special person to facilitate calculation) and $\left|(S P+O N K)_{k e k}\right|$ represents the total number of persons and $O N K$ who contain $k e_{k}$.

After calculating the weight of each knowledge element, $P_{i}$ and $O N K$ can be expressed by weight vector as follows: $P_{i}($ or $O N K)=\vec{p}_{i}$ (or $\left.\overrightarrow{o n k}\right)=\left(w_{i k}\right)_{1 \times n}=\left[w_{i 1}, w_{i 2}, \ldots, w_{i n}\right]$. Consequently, the similarity between $P_{i}$ and $O N K$ computed by the vector cosine $[16,17]$ is $\operatorname{SIM}\left(P_{i}\right.$, ONK $)=\cos \left(\vec{p}_{i}, \overrightarrow{\text { onk }}\right)=\frac{\sum_{k=1}^{n} w_{i k} \times w_{j k}}{\sqrt{\left[\left(\sum_{k=1}^{n} w_{i k}^{2}\right)\left(\sum_{k=1}^{n} w_{j k}^{2}\right)\right]}}$.

Based on the above procedure, Yu et al. [3] proposed that the person with the maximum similarity should be selected to train. However, Yu et al. [3] did not formulate the person selection of organization personnel training, which results in lack of theoretical analysis. Therefore, section 3.1 summarizes the main contributions of [3] and formulates the mathematical model.

\section{Models for Selecting Single Person and Selecting Multiple Persons}

\subsection{Model for Selecting Single Person}

3.1.1. Index: Index $i$ refers to the index of persons $(i=1,2, \ldots,|S P|)$, where $|S P|$ is the total number of persons.

3.1.2. Parameters: $O N K$ : Organization needing knowledge;

$P_{i}:$ Knowledge of person $i$

$S P$ : Set of persons;

SIM $\left(O N K, P_{i}\right)$ : Similarity between organization needing knowledge and the personnel's knowledge and can be calculated by the method in section 2.2.6.

3.1.3. Decision Variable: $X_{i}=\left\{\begin{array}{l}1, \text { if person } i \text { is selected; } \\ 0 \text {, otherwise. }\end{array}\right.$

\subsubsection{Objective Function:}

$$
N \operatorname{sim}=\max \sum_{i=1}^{|S P|} \operatorname{SIM}\left(\text { ONK }, P_{i}\right) \times X_{i}
$$

It subjects to

$$
\sum_{i=1}^{|S P|} X_{i}=1
$$

3.1.5. Features: Obviously, the complexity of the model is $|S P|$, because the person with the maximum similarity is selected to train. In other words, the model is to select only one person to train. However, it is necessary to select multiple persons simultaneously in organization personnel training. Accordingly, we propose the model for selecting multiple persons.

\subsection{Model for Selecting Multiple Persons}

3.2.1. Formulation: Different from the constraint in the model for selecting single person, the constraint in the model for selecting multiple persons is: 


$$
\sum_{i=1}^{|S P|} X_{i}=M
$$

In the formula, $M$ denotes the number of persons to be trained. $M=1$ means only one person is selected, i.e., the model for selecting single person. $M>1$ means multiple persons need to be selected. Therefore, the model has better applicability than the model for selecting single person.

3.2.2. Features: Multiple persons are selected by the model. For example, in a case with $|S P|$ persons, 3 persons are selected to train. The similarity set of $\left\{\operatorname{SIM}\left(O N K, P_{i}\right) \mid\right.$ $i=1,2, \ldots,|S P|\}$ will be sorted in decreasing order. Subsequently, the first 3 persons with the maximum similarity are selected to train.

The complexity of the model is determined by the algorithm of sorting $\{$ SIM (ONK, $\left.\left.P_{i}\right)|i=1,2, \ldots| S P \mid,\right\}$. If bubble sort is used, then the complexity is $|S P|^{2}$. If quick sort is used, then the complexity is $|S P| \lg |S P|$.

3.2.3. Shortcoming: Although the model is better than the model for selecting single person, it may produce unreasonable results. For example, in the case that $O N K$ contains two fields and the first 3 maximum similarities are: $0.8,0.7$, and 0.6. The first two persons (assume they are person 1 and person 2) belong to a field and the third person (assume person 3) belongs to another field. If selecting one person, then obviously person 1 should be selected. This is consistent with the result of Yu et al. [3]. If selecting two persons to supplement knowledge of the two fields simultaneously, then obviously person 1 and person 3 should be selected. However, using the model for selecting multiple persons, persons 1 and 2 will be selected. This is unreasonable. That is because is the model does not consider the change of organization needing knowledge after selecting person 1. In fact, after selecting person 1, organization needing knowledge has been supplemented partly. Subsequently, organization needing knowledge $(O N K)$ should be expressed as $O N K-P_{1}$

\section{Model for Selecting Multiple Persons based on Step-by-step Method}

Based on the idea that organization needing knowledge changes after one person is selected, we propose the model for selecting multiple persons based on step-by-step method.

\subsection{Notations}

4.1.1. Indices: $m$ : Index of steps $(n=1,2, \ldots, M)$;

$i$ : Index of persons who is not selected $(i=1,2, \ldots,|S U P|)$.

4.1.2. Parameters: $O N K_{1}$ : Organization needing knowledge of the first step;

$S S P_{1}$ : Set of selected persons of the first step, null set;

$S U P_{1}$ : Set of unselected persons of the first step, containing all persons;

$U P_{i}$ : Knowledge of unselected person $i$;

$\operatorname{SIM}\left(O N K_{1}, U P_{i}\right)$ : Similarity between $O N K_{1}$ and $U P_{i}$.

4.1.3. Variables: $O N K_{m}$ : Organization needing knowledge of the $m^{\text {th }}$ step;

$S S P_{m}$ : Set of selected persons of the $m^{\text {th }}$ step, $m-1$ persons in $S S P_{m}$;

$S U P_{m}$ : Set of unselected persons of the $m^{\text {th }}$ step;

$\operatorname{SIM}\left(O N K_{m}, U P_{i}\right)$ : Similarity between $O N K_{m}$ and $U P_{i}$. 
4.1.4. Decision Variable: $X_{m i}=\left\{\begin{array}{l}1, \text { if unselected person } i \text { is selected in step } m \text {; } \\ 0 \text {, otherwise. }\end{array}\right.$

\subsection{Formulation of Selecting Multiple Persons based on Step-by-step Method}

Objective function:

$$
N \operatorname{sim}=\max \sum_{m=1}^{M} \sum_{i=1}^{\left|S U P_{m}\right|} \operatorname{SIM}\left(O N K_{m}, U P_{i}\right) \times X_{m i}
$$

It subjects to:

$$
\begin{gathered}
\sum_{i=1}^{\left|S U P_{m}\right|} X_{m i}=1 \forall m \\
\sum_{m=1}^{M} \sum_{i=1}^{\left|S U P_{m}\right|} X_{m i}=M \\
\sum_{m=1}^{M} X_{m i} \leq 1 \quad \forall i \\
O N G_{m}=O N G_{(m-1)}-\sum_{i=1}^{\left|S U P_{(m-1)}\right|} U P_{i} \times X_{(m-1) i} \\
S U P_{m}=S U P_{(m-1)}-\sum_{i=1}^{\left|S U P_{(m-1)}\right|} U P_{i} \times X_{(m-1) i} \\
S S P_{m}=S S P_{(m-1)}+\sum_{i=1}^{\left|S U P_{(m-1)}\right|} U P_{i} \times X_{(m-1) i}
\end{gathered}
$$

Equation (9) means the person with the maximum similarity in step $m$ should be selected. Equation (10) is the constraint that guarantees only one person is selected in each step. Equation (11) is the constraint of total number of selected persons, i.e., $M$ persons are selected. Equation (12) ensures that each person is selected at most one time. Equation (13) expresses the new $O N K$ after selecting one person. Equation (14) expresses the set of unselected persons after selecting a person. Equation (15) expresses the set of selected persons after selecting one person.

\subsection{Complexity}

In step $m$, there are $\left|S U P_{m}\right|$ persons, and so the complexity is $C_{|S U P m|}{ }^{1}$. Therefore, the complexity of selecting $M$ persons is $P_{|S U P m|}{ }^{M}=\prod_{m=1}^{M}\left|S U P_{m}\right|$. If $M=1$, then the model equals the model for selecting single person. If $M>1$, then the model is a combinational problem. Obviously, the complexity of the model is much larger than that of the model for selecting multiple persons.

\section{Computational Experiments}

\subsection{Test Instance}

Suppose that a knowledge-intensive organization wants to improve the knowledge structure by personnel training. The organization has 3 persons and 38 files containing personnel knowledge. To verify our proposed model, we select 2 persons from the three persons to train. Test data are shown in Tables 1 and 2. 
Table 1. Data of Unselected Persons

\begin{tabular}{ccc}
\hline $\begin{array}{c}\text { Unselected } \\
\text { Person }\end{array}$ & Field of personnel knowledge & $\begin{array}{c}\text { Number of } \\
\text { documents }\end{array}$ \\
\hline 1 & $\begin{array}{c}\text { Management science and } \\
\text { engineering } \\
\text { Management science and } \\
\text { engineering }\end{array}$ & 15 \\
3 & Business management & 13 \\
\hline
\end{tabular}

Table 2. Similarities between $O N K_{1}$ and the Unselected Persons

\begin{tabular}{cc}
\hline Unselected Person & $\operatorname{SIM}\left(\mathrm{ONK}_{1}, U \mathrm{UP}_{i}\right)$ \\
\hline 1 & 0.8 \\
2 & 0.7 \\
3 & 0.6 \\
\hline
\end{tabular}

\subsection{Result of Selecting Wholly Persons}

Using the model of selecting wholly multiple persons, person 1 and person 2 will be selected. However, both person 1 and person 2 supplement the knowledge of management science and engineering. Therefore, the organization needing knowledge in business management cannot be supplemented. This is unreasonable.

\subsection{Result of Selecting Persons based on Step-by-step Method}

By using the model for selecting multiple persons based on step-by-step method, person 1 will be selected in the first step. Subsequently, the new organization needing knowledge is modified as $O N K_{1}-U P_{1}$. Then $\operatorname{SIM}\left(O N K_{2}, U P_{i}\right)$ will be calculated. The result is shown in Table 3.

Table 3. Similarities between $O N K_{2}$ and Unselected persons

\begin{tabular}{cc}
\hline Unselected Person & $\operatorname{SIM}\left(\mathrm{ONK}_{2}, \mathrm{UP}_{i}\right)$ \\
\hline 2 & 0.4 \\
3 & 0.8
\end{tabular}

Based on the result of Table 3, person 3 will be selected in the second step. Thus, the two fields of both management science and engineering and business management can be supplemented.

The reason of $\operatorname{SIM}\left(\mathrm{ONK}_{2}, \mathrm{UP}_{2}\right)<\operatorname{SIM}\left(\mathrm{ONK}_{2}, U P_{3}\right)$ is because person 1 has supplemented the knowledge in management science and engineering. Consequently, the knowledge in business management is in the great need of organization.

\section{Conclusions and Future Research}

Our contributions in this paper can be summarized as following: Firstly, we formulate the model for selecting single person. Subsequently, we propose the model for selecting multiple persons and clarify the complexity and the features. Finally, we propose the model for selecting multiple persons based on step-by-step method to improve the accuracy of personnel selection.

The shortcomings or the features of the three proposed models are summarized in Table 4. The complexities of the three proposed models are summarized in Table 5. 
Table 4. Shortcomings or Features of Three Proposed Models

\begin{tabular}{|c|c|}
\hline Models & Shortcomings or features \\
\hline Model of selecting single & Single person selection \\
\hline $\begin{array}{l}\text { Model of selecting wholly multiple } \\
\text { persons }\end{array}$ & Multiple persons selection, unreasonable result \\
\hline $\begin{array}{l}\text { Model of selecting multiple persons } \\
\text { based on step-by-step method }\end{array}$ & $\begin{array}{c}\text { Multiple persons selection, reasonable result, and } \\
\text { higher complexity }\end{array}$ \\
\hline
\end{tabular}

Table 5. Complexities of three Proposed Models

\begin{tabular}{lcc}
\hline \multicolumn{1}{c}{ Models } & $\begin{array}{c}\text { Number of selected } \\
\text { persons }\end{array}$ & Complexity \\
\hline Model of selecting single person & 1 & $|S P|$ \\
Model of selecting wholly multiple & 1 & $|S P|$ \\
persons & $>1$ & Up to the algorithm to sort, \\
& 1 & such as $|S P|^{2}$ and $|S P| l g|S P|$ \\
$C_{|S U P m|}($ i.e., $|S P|)$ \\
$\begin{array}{l}\text { Model of selecting multiple persons } \\
\text { based on step-by-step method }\end{array}$ & $>1$ & $P_{|S U P m|}{ }^{M}=\prod_{m=1}^{M}|S U P m|$ \\
\hline
\end{tabular}

$|S P|$ is the total number of persons. $\left|S U P_{1}\right|$ is the number of unselected persons in the first step and equals to $|S P|$. $\left|S U P_{m}\right|$ is the number of unselected persons in step $m$.

However, there are still some shortcomings in the proposed model for selecting multiple persons based on step-by-step method. For example, not only higher similarity with organization needing knowledge needs to be considered in selecting person, other aspects, such as current capacity, potential, character, attitude, and gender also should be concerned as well. In addition, the complexity of the model for selecting multiple persons based on step-by-step method is high. The effective method for rapidly selecting persons needs to be researched in the future.

\section{Acknowledgments}

The authors would like to thank the anonymous referee and the editor for their careful consideration and valuable comments.

This research is supported by the National Natural Science Foundation of China (61203182), the National Social Science Foundation of China (13CGL045), and Liaoning Education Science Program (JG15EB061).

\section{References}

[1] L. B. Chi-ang, "A sustainable perspective on the knowledge economy: a critique of Austrian and mainstream views", Ecol. Econ., vol. 60, no. 1, (2006).

[2] Z. Wang, B. He and N. Li, "Solution of the learning organization's introducing knowledge and talents based on indifference analysis", Sci. Tech. Manag. Res., vol. 27, no. 10, (2007).

[3] Y. Yu and Y. Z. Dang, "Supernetwork model of personnel training in organization", Syst. Eng. -Theor. Pract., vol. 29, no. 4, (2009).

[4] L. Zhang, R. Yang and F. Mei, „Model discussion of personal training and hi-tech commercializing in universities", Journal Univ. Electron. Sci. Tech. China, vol. 30, no. 3, (2001).

[5] Q. Yao and S. Zhao, "On training university students in scientific outlook", Journal Northwest Univ., vol. 37, no. 6, (2007).

[6] R. Song and Z. Pei, "Strategies on the recruitment of talents in universities", Tsinghua J. Educ., vol. 24, no. 6, (2003).

[7] G. Zuo, "The application of AHM method for the qualified personnel introduce", Math. Pract. Theor., vol. 36, no. 9, (2006). 
[8] A. Nagurney and J. Dong, "Management of knowledge intensive systems as supernetworks: modeling, analysis, computations, and applications", Math. Comput. Model, vol. 42, no. 3-4, (2005).

[9] Y. Yu, "Researches on knowledge supernetwork in organizational knowledge management", $\mathrm{PhD}$ Thesis. Dalian Univ. Technol. China, (2009).

[10] Y. J. Xi, Y. Z. Dang and K. J. Liao, "Knowledge supernetwork model and its application in organizational knowledge systems", Journal Manage. Sci. China, vol. 12, no. 3, (2009).

[11] Y. J. Xi and Y. Z. Dang, "The method to analyze the robustness of knowledge network based on the weighted supernetwork model and its application”, Syst. Eng. -Theor. Pract., vol. 27, no. 4, (2007).

[12] W. Sun, Q. H. Ma and Y. Yu, "Personnel selection model of innovation team based on knowledge supernetwork, Sci. Sci. Manage. S. \& T., vol. 34, no. 8, (2013).

[13] W. Sun, Q. H. Ma and Y. Yu, "Multi-objective model for talent recruiting and its theoretical analysis from knowledge perspective", Journal Northeastern Univ., vol. 33, no. 8, (2012).

[14] W. Sun and Y. Yu, "Staff similarity computation in technology innovation team", Adv. Mater. Res., (2011), pp. 204-210.

[15] Z. G. Xuan, "Text mining algorithm and its application in knowledge management", PhD Thesis, Dalian Univ. Technol., China, (2008).

[16] G. Salton and C. Yang, "On the specification of term values in automatic indexing", Journal Doc., vol. 29, no. 4, (1973)

[17] G. Salton, A. Wong and C. Yang, "A vector space model for automatic indexing", Comm. ACM., vol.18, no. 11, (1975).
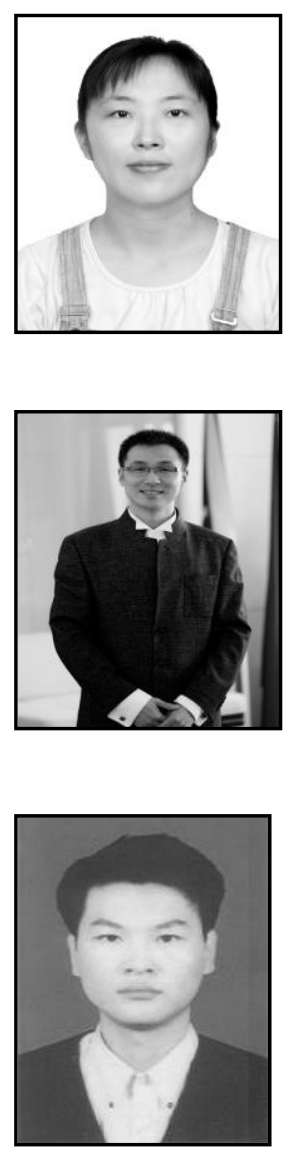

\section{Authors}

Wei Sun, she received Ph.D. degree in management science and engineering, Dalian University of Technology, Dalian, China, in 2008. From December 2008 onwards she has been serving as an associate professor in the Business School, Liaoning University, Shenyang, China. Her major researches include complex network, Management Science, Operations Research, etc.

Chunhui Huo, he received Ph.D. degree in management science, Liaoning University, Shenyang, China, in 2006. From 2007 onwards he has been serving as a faculty member in Business School, Liaoning University. His major researches include Strategic Management, Management Science, etc.

Yang Yu, he received Ph.D. degree in management science and engineering, Dalian University of Technology, Dalian, China, in 2009. From 2009 June onwards he has been serving as a faculty member in Information Science and Engineering School, Northeastern University, Shenyang, China. His major researches include Complex Network, Supernetwork, Operations Research, Management Science. 
International Journal of Future Generation Communication and Networking Vol. 9, No. 12 (2016) 